\title{
The Usage and Efficiency of Drug- Eluting Stents in Vertebral Ostial Stenosis
}

\author{
Burcu Erkan' (iD , Serdar Geyik² (D) , Kıvılcım Yavuz ${ }^{3}$ (D) , Işıl Saatçi ${ }^{4}$ iD , \\ Saruhan Çekirge ${ }^{4}$ iD
}

${ }^{1}$ Koç Üniversitesi Tıp Fakültesi, Radyoloji Anabilim Dalı, İstanbul, Türkiye

${ }^{2}$ İstanbul İstinye Üniversitesi Tıp Fakültesi, Radyoloji Anabilim Dalı, İstanbul, Türkiye

${ }^{3}$ Ankara Üniversitesi Tıp Fakültesi, Radyoloji Anabilim Dalı, Ankara, Türkiye

${ }^{4}$ Yüksek Ihtisas Üniversitesi Koru Hastanesi, Radyoloji Anabilim Dalı, Ankara, Türkiye

Burcu ERKAN, Dr. Öğr. Üyesi

Serdar GEYIK, Prof. Dr.

Kivilcım YAVUZ, Prof. Dr.

IşıI SAATÇI, Prof. Dr.

Saruhan ÇEKIRGE, Prof. Dr.

Correspondence: Burcu Erkan

Koç Üniversitesi Tıp Fakültesi, Radyoloji

Anabilim Dalı, İstanbul, Türkiye

Phone: +905326528206

E-mail: bakpinar@kuh.ku.edu.tr

Received

\section{ABSTRACT}

Objectives: Extracranial vertebral artery atherosclerosis is an insidious and hazardous disease. With technological development and accumulating experience, antiproliferative drug-eluting stents became a viable option for reducing the in-stent restenosis of the origin of the vertebral artery. Here, we evaluated the technical success rates, efficiency, clinical and angiographic results of the usage of drugeluting stents in vertebral ostial stenosis.

Patients and Methods: 28 stents were implanted in 24 patients with vertebral artery origin stenosis. Digital subtraction angiographic or CT angiographic follow-up was made at 6, 12 and 24 months.

Results: Paclitaxel-eluting stents were placed with high technical success for the treatment of vertebral artery origin stenosis. There was no procedure-related mortality. However, one patient succumbed to death due to aspiration pneumonia for a basilar artery stroke with successful stenting and thrombolysis procedure. There was only one limited subclavian artery dissection in a patient (\%4.1) during the procedure which was managed conservatively. One stent (\%3.7) had in-stent restenosis in the early period (6th month) and one patient (\%4.1) had recurrent neurological symptoms on follow-up (9th month). In a median follow-up of 13 months (6-25 months), none of the patients had late stent thrombosis.

Conclusion: Vertebral artery ostial stenosis can be treated effectively and safely with high technical success and low in-stent restenosis rates with paclitaxel drug-eluting stents. With low restenosis rates, antiproliferative drug-eluting stents are an option for reducing the vertebral artery in-stent restenosis.

Keywords: Vertebral artery origin, stenosis, paclitaxel, drug-eluting stent

\section{Vertebral Ostial Stenoz Tedavisinde Illaç Salınımlı Stent Uygulaması ve Etkinliği}

ÖZET

Amaç: Ekstrakraniyal vertebral arter aterosklerotik hastalığı sinsi ve tehlikeli bir hastalıktır. Gelişen teknoloji ve artan tecrübelerle birlikte antiproliferatif ilaç salınımlı stentler vertebral arter orjininde stent içi stenozu azaltmaya alternatif oluşturmaktadır. Biz de çalışmamızda vertebral ostial stenozlarda ilaç salınımlı stent uygulamasının teknik başarıııı, etkinliğini, klinik ve anjiyografik sonuçlarını değerlendirdik.

Hastalar ve Yöntemler: Vertebral arter orijin darlı̆ı olan 24 hastaya toplam 28 adet stent yerleştirildi. 6, 12, 24. aylarda anjiyografi veya BT anjiyografi ile takip edildi.

Bulgular: Vertebral arter orijin darlıklarının tedavisinde paclitaxel salııımlı stent yüksek teknik başarı ile uygulandı. İ̧leme bağı mortalite izlenmedi. Baziler arter inmesi nedeniyle başarlı stentleme ve tromboliz uygulanmış bir olgu aspirasyon pnömonisi nedeniyle kaybedildi. İ̧̧leme bağlı bir hastada (\%4.1) subklavyen arterde sınırlı diseksiyon geliş̧i ve medikal tedavi ile takip edildi. Takipte bir adet stentte (\%3.7) erken dönem (6.ay kontrol) stent içi restenoz gelişti, bir hastada (\%4.1) rekürren nörolojik semptom izlendi (9.ay). Hastaların hiçbirinde geç dönem tromboz izlenmedi.

Sonuç: Vertebral arter ostial stenozlarında ilaç salınımlı stentler yüksek teknik başarı ve düşük stent içi restenoz oranları ile etkin ve güvenle kullanılabilir. Düşük restenoz oranları ile de antiproliferatif ilaç salınımlı stentler VA orjininde stent içi stenozu azaltmaya alternatif oluşturmaktadır.

Anahtar Sözcükler: Vertebral arter orijin, stenoz, paclitaxel, ilaç salınımlı stent 
V ertebrobasilar infarcts are $25 \%$ of all cerebral infarcts (1). 5-year recurrent stroke rate is reported to be about $22-35 \%$ after a vertebrobasilar transient ischemic attack (TIA) or stroke (2). Ostium is the most common place for vertebral artery (VA) stenosis (3).

Medical treatment is classically the initial treatment in VA stenosis (4). In cases where medical treatment is inadequate, angioplasty and stenting are preferred options for symptomatic vertebrobasilar atherosclerotic disease to avoid surgical-related morbidity (4-6). Primary stenting with balloon-expandable coronary stents is reported to be applied safely with high technical success in VA origin stenosis (OS). Nevertheless, relative in-stent restenosis (ISR) is still a problem to be solved. Antiproliferative drugeluting stents (DES) offer an alternative for decreasing the ISR in VA origin. In our study, we assessed the technical success, efficiency, clinical and angiographic results of DESs in vertebral ostial stenosis.

\section{Methods}

The institutional review board approved this retrospective study and waived informed consent.

\section{Patient Information}

Patients who had VAOS with percutaneous endovascular Paclitaxel eluting stenting procedure in our institution between 2006 to 2008 were included in this retrospective study.

Age, gender, medical histories, clinical findings of the subjects and administered stent diameter were collected from medical records.

\section{Stenting procedure}

Endovascular treatment was indicated for the patients that had vertebrobasilar insufficiency or a history of TIA or stroke with 50\% and higher VAOS which was determined by vertebral angiography. VAOS rates were measured with modified NASCET (North American Symptomatic Carotid Endarterectomy Trial) criteria (7), which was used for carotid stenosis, using diagnostic digital subtraction angiograms (DSA). All patients had pre and post-neurological examinations. Before the procedure, all patients were informed of the procedure and complications, and all patients signed the informed consent form. All procedures were performed by experienced interventional neuroradiologists in a DSA system (Artis, Siemens Medical Solutions, Erlangen, Germany).

In our procedure, all elective patients were medicated with clopidogrel bisulfate $75 \mathrm{mg} /$ day, acetylsalicylic acid (ASA) $300 \mathrm{mg} /$ day, starting 5 days before the procedure. In emergency situations, $300 \mathrm{mg}$ clopidogrel bisulfate and $300 \mathrm{mg}$ ASA were administered before the procedure as a loading dose.

During the procedure after $70-100 \mathrm{U} / \mathrm{kg}$ bolus heparin infusion, 7- $10 \mathrm{U} / \mathrm{kg} / \mathrm{hour}$ heparin infusion was given to keep the activated clotting time level between 250-300 seconds.

Stent diameter was chosen according to the distally normal VA diameter, stent length was chosen to cover the entire atherosclerotic plaque.

After the procedure, we recommended ASA, $100 \mathrm{mg} /$ day, lifelong, and clopidogrel, $75 \mathrm{mg} /$ day, for two years.

In our clinic, we keep the patients under a control schedule at 6,12, 24 months. Stents were evaluated with DSA at $6^{\text {th }}$ and $24^{\text {th }}$ months, with computed tomography angiography (CTA) at $12^{\text {th }}$ months.

\section{Evaluation}

The location of the lesion and stenosis rates were reviewed from the pre-procedure DSA. Stenting procedure of the vertebral ostial stenosis and other necessary locations, additional findings and complications were reviewed and noted. The pre and post-procedural Magnetic Resonance imaging (MRI) were reviewed if available.

Follow-up DSA and CTA findings were evaluated from Picture Archiving and Communication System (PACS ${ }^{\oplus}$ ) and noted retrospectively. For CTA datasets, the axial $0.5 \mathrm{~mm}$ thin slices, coronal and sagittal reformatted images with maximum intensity projections obtained by dual source CT scanner (SOMATOM Definition, Siemens Medical Solutions, Erlangen, Germany) were reviewed. Hemodynamically significant ISR is considered in $>50 \%$ stenosis. Morbidity and mortality rates as well as restenosis rates in 6,12 and 24 months were noted. 


\section{Results}

Four patients were lost to follow-up after discharge, so they were excluded from the final analysis. Twenty-four patients (M: $F=20: 4$, mean age 60 , range 47-81) who had VAOS with a total of 28 Paclitaxel eluting stents (PES) (Taxus- Boston Scientific) implanted by percutaneous endovascular procedure, were included in the study.

The majority of the patients were referred to our clinic from neurology clinics for a previous cerebrovascular event or persistent ischemic symptoms of posterior circulation despite optimal medical treatment. Three patients were diagnosed during cardiovascular evaluations (case $8,10,23)$.

All patients' medical histories were investigated and summarized in table 1.22 patients had a history of TIA, stroke or vertebrobasilar insufficiency. Nine patients had a previous stroke history (3 of them from posterior circulation) and 2 patients had cerebrovascular events meanwhile. Basically, vertebrobasilar insufficiency was evaluated by clinical information. Four patients had pre-procedural CTA.

There were 2 asymptomatic patients, diagnosed during the cardiovascular work-up (case 10) and angiography investigation for the leg angina (case 8). These patients were treated for the diagnosis of high stenosis (>90\%) rates of the VA with accompanying cerebrovascular occlusions and/or stenosis.

Nineteen patients had pre-procedural MRI and 3 of them (case $6,11,17$ ) had acute ischemic lesions $(15.7 \%)$.
The VAOS rates were subclassified as $>90 \%(n=13), 90-$ $70 \%(n=8)$ and $69-50 \%(n=6)$ when measured with modified NASCET. There was one unclassified intervention which was a broken stent (case 12), previously installed in another institution.

Nineteen patients had predisposing factors for atherosclerotic disease (79.1\%). Nine patients had one or more findings of hemiparesis, loss of sensation, cerebellar ataxia, dysphasia, during the neurological examination (37.5\%).

In our study, all vessels were stented successfully with the percutaneous endovascular method. During the stenting procedure, none of the lesions needed predilatation balloon angioplasty. A DSA example of a case with pre and post-stenting images is shown in Fig. 1.

After the procedure, none of the control angiograms showed either an intracranial missing branch or an intraluminal filling defect suggestive of distal embolization. One patient (case 19) among 24 patients (4.1\%) was diagnosed with a limited proximal subclavian artery dissection on post-procedure control angiogram. The patient was only anticoagulated without additional intervention. One week later the control CTA revealed the findings as stable.

None of the patients developed permanent neurological symptoms or any deficit on post-procedure neurological examinations. Two patients with temporary neurological symptoms had post-procedural MRI performed. One patient with a complaint of numbness on the left hand developed two millimetric lesions in the right postcentral gyrus with restricted diffusion (case 3 ) and another patient with the clinical finding of emotional indifference-agitation had a millimeter sized hemorrhagic lesion in the corpus of the left caudate nucleus (case 6). The clinical findings of both were disappeared during early follow-up (within one week).

Fig. 1: A DSA example of a case with pre (a) and post-stenting (b) images. The left VA origin stenosis was treated with a paclitaxel-eluting stent.
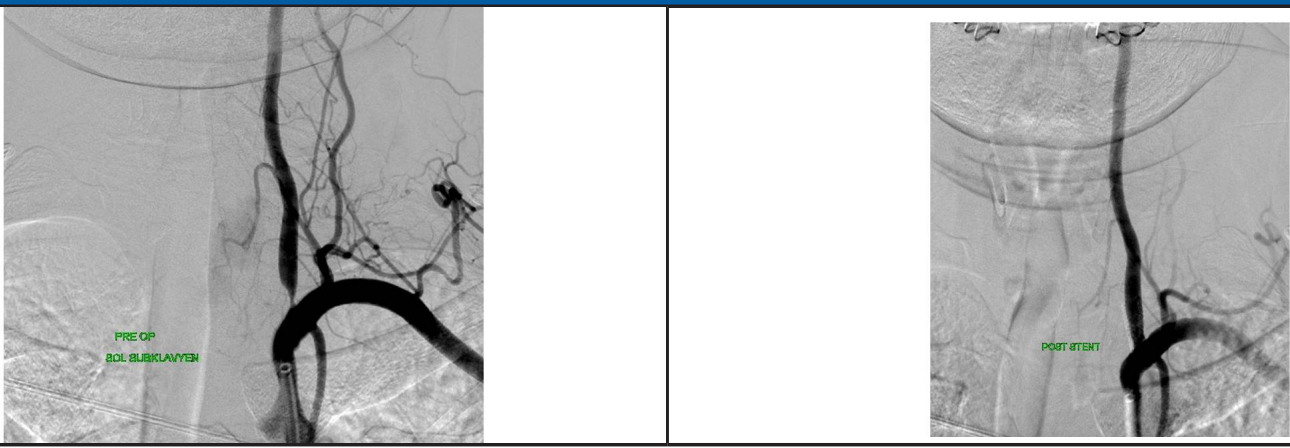


\begin{tabular}{|c|c|c|c|c|c|c|c|c|c|c|c|c|c|c|c|}
\hline $\begin{array}{c}\text { Case } \\
\text { No }\end{array}$ & Sex & Age & Symptoms & $\begin{array}{l}\text { Clinical } \\
\text { Findings }\end{array}$ & $\begin{array}{l}\text { Imaging } \\
\text { finding-pre }\end{array}$ & $\begin{array}{l}\text { Imaging } \\
\text { findings-post }\end{array}$ & $\begin{array}{l}\text { Comor } \\
\text { bidity }\end{array}$ & $\begin{array}{l}\text { Lesion } \\
\text { location }\end{array}$ & $\begin{array}{c}\text { Stenosis } \\
\%\end{array}$ & $\begin{array}{l}\text { Stent } \\
\text { Taxus } \\
(\mathrm{mm})\end{array}$ & $\begin{array}{c}\text { 6th } \\
\text { Month } \\
\text { Control } \\
\text { Angio }\end{array}$ & $\begin{array}{c}1 \text { st } \\
\text { Year } \\
\text { Control } \\
\text { CTA }\end{array}$ & $\begin{array}{l}\text { 2nd } \\
\text { Year } \\
\text { control } \\
\text { Angio }\end{array}$ & $\begin{array}{l}\text { Other } \\
\text { stented } \\
\text { area/ } \\
\text { stenosis } \\
\text { Additional } \\
\text { findings }\end{array}$ & Complication \\
\hline 1 & W & 53 & $\mathrm{~V}, \mathrm{NV}$, At & - & $\begin{array}{l}\text { MRI: No acute } \\
\text { ischemic } \\
\text { lesion. A } \\
\text { couple } \\
\text { millimetric } \\
\text { nonspesific } \\
\text { lesion }\end{array}$ & - & $\mathrm{Ht}$ & $\begin{array}{l}\text { RVA } \\
\text { LVA }\end{array}$ & $\begin{array}{l}70-90 \\
70-90\end{array}$ & $\begin{array}{l}2.5 \times 12 \\
4.5 \times 12\end{array}$ & $\begin{array}{c}\mathrm{P} \\
\mathrm{MIH}\end{array}$ & $\begin{array}{l}P \\
P\end{array}$ & $\begin{array}{c}P \\
M I H\end{array}$ & - & - \\
\hline 2 & M & 66 & $\begin{array}{c}\mathrm{V}, \mathrm{Hd}, \mathrm{Wk} \\
\text { temporary } \\
\mathrm{D}, \mathrm{Di}\end{array}$ & - & - & - & $\begin{array}{c}\mathrm{Ht}, \\
\mathrm{DM}, \mathrm{HL}, \\
\mathrm{C}\end{array}$ & LVA & $50-70$ & $5 \times 12$ & $P$ & - & $\mathrm{MIH}$ & $\begin{array}{c}\text { LICA } \\
\% 50-70 \\
\text { L Renal A }\end{array}$ & - \\
\hline 3 & W & 48 & $\mathrm{Vd}$ & - & $\begin{array}{l}\text { MRI: A couple } \\
\text { milimetric } \\
\text { chronic } \\
\text { ischemic } \\
\text { lesion }\end{array}$ & $\begin{array}{c}\text { MRI: } \\
\text { Peripheral } \\
\text { and median } \\
\text { milimetric } \\
\text { acute } \\
\text { ischemic } \\
\text { lesion on R } \\
\text { postcentral } \\
\text { gyrus }\end{array}$ & $\begin{array}{c}\mathrm{DM}, \mathrm{Ht}, \\
\mathrm{RA}, \mathrm{Hs}\end{array}$ & $\begin{array}{l}\text { RVA } \\
\text { LVA }\end{array}$ & $\begin{array}{l}>90 \\
>90\end{array}$ & $\begin{array}{c}4 \times 12 \\
4.5 \times 16\end{array}$ & $\begin{array}{l}\text { MIH } \\
\text { MIH }\end{array}$ & $\begin{array}{l}P \\
P\end{array}$ & $\begin{array}{l}P \\
P\end{array}$ & $\begin{array}{c}\text { R ICA }>\% 90 \\
\text { (L ICA } \\
\text { stenosis- } \\
\text { follow-up) }\end{array}$ & - \\
\hline 4 & M & 61 & Vd, L Hp & $\begin{array}{c}\text { (Previous } \\
\text { CVE) } \\
\text { LUE } 3 / 5 \text {, LLE } \\
4 / 5\end{array}$ & $\begin{array}{c}\text { MRI: Chronic } \\
\text { ischemic lesion } \\
\text { on R putamen, } \\
\text { centrum } \\
\text { semiovale } \\
\text { Wallerian } \\
\text { degeneration, } \\
\text { large R lateral } \\
\text { ventricule }\end{array}$ & - & - & LVA & $50-70$ & $5 \times 12$ & $P$ & $\mathrm{MIH}$ & - & $\begin{array}{c}\text { LICA } \\
\% 70-90 \\
\text { (R subclav, } \\
\text { brakiocef } \\
\text { occlusion, } \\
\text { steal) } \\
\text { (L subclav } \\
\text { stenosis- } \\
\text { follow-up) }\end{array}$ & - \\
\hline 5 & M & 60 & $\mathrm{RH}$ & $\begin{array}{l}\text { (Previous } \\
\text { CVE- L PCA) } \\
\text { Loss of } \\
\text { sensation } \\
\text { Incompetant } \\
\text { cerebellar } \\
\text { examination }\end{array}$ & $\begin{array}{l}\text { MRI: Chronic } \\
\text { ischemic } \\
\text { lesion on } \\
\text { left occipital, } \\
\text { posterior } \\
\text { talamus, } \\
\text { bilateral } \\
\text { cerebellar }\end{array}$ & - & HT & LVA & $50-70$ & $4.5 \times 12$ & $\mathrm{MIH}$ & $P$ & - & $\begin{array}{c}\text { (Hypoplastic } \\
\text { RVA) } \\
\text { (L ICA } \\
\text { stenosis- } \\
\text { follow-up }\end{array}$ & - \\
\hline 6 & $M$ & 48 & L Hp & $\begin{array}{l}\text { (Previous } \\
\text { CVE) } \\
\text { LUE } 4 / 5\end{array}$ & $\begin{array}{l}\text { MRI: RMCA } \\
\text { area large } \\
\text { chronic infarct, } \\
\text { volume loss. } \\
\text { Front nearby- } \\
5 \mathrm{~mm} \text { acute } \\
\text { ischemic } \\
\text { lesion }\end{array}$ & $\begin{array}{c}\text { MRI: } \\
\text { Milimetric } \\
\text { new } \\
\text { hemorrhagic } \\
\text { lesion on the } \\
\text { corpus of } \\
\text { left caudat } \\
\text { nucleus }\end{array}$ & - & RVA & $70-90$ & $3.5 \times 12$ & $p$ & $p$ & $p$ & $\begin{array}{c}\text { R ICA }>\% 90 \\
\text { LICA } \\
\% 70-90\end{array}$ & - \\
\hline 7 & $M$ & 54 & L Hp, Fp & $\begin{array}{l}\text { (Previous } \\
\text { CVE) } \\
\text { Indistinct L } \\
\text { nasolabial } \\
\text { sulcus, } \\
\text { LUE 3/5, LLE } \\
4 / 5 \\
\text { L } \\
\text { hyperactive } \\
\text { DTR }\end{array}$ & $\begin{array}{c}\text { MRI: Chronic } \\
\text { ischemic } \\
\text { gliotik lesion } \\
\text { on R temporal } \\
\text { lobe } \\
\text { CTA: Saccular } \\
\text { aneurism on } \\
\text { arcus aorta }\end{array}$ & - & C & LVA & $>90$ & $4.5 \times 12$ & $\mathrm{MIH}$ & $\mathrm{p}$ & $\mathrm{p}$ & $\begin{array}{c}\text { R ICA >\%90 } \\
\text { (RVA } \\
\text { stenosis- } \\
\text { follow-up) }\end{array}$ & - \\
\hline 8 & $M$ & 70 & EA & - & $\begin{array}{l}\text { MRI: Chronic } \\
\text { ischemic } \\
\text { gliotic lesions } \\
\text { on bilateral } \\
\text { centrum } \\
\text { semiovale, } \\
\text { posterior } \\
\text { periventricular } \\
\text { area }\end{array}$ & - & CAD & $\begin{array}{l}\text { RVA } \\
\text { LVA }\end{array}$ & $\begin{array}{l}>90 \\
>90\end{array}$ & $\begin{array}{c}4 \times 12 \\
4.5 \times 12\end{array}$ & $\begin{array}{c}\mathrm{P} \\
\mathrm{MIH}\end{array}$ & $\begin{array}{l}\mathrm{P} \\
\mathrm{P}\end{array}$ & $\begin{array}{c}\mathrm{MIH} \\
\mathrm{P}\end{array}$ & $\mathrm{R}$ ICA $>\% 90$ & - \\
\hline
\end{tabular}




\section{Table 1. Patients demographics, findings and treatments (continued)}

\begin{tabular}{|c|c|c|c|c|c|c|c|c|c|c|c|c|c|c|c|}
\hline $\begin{array}{l}\text { Case } \\
\text { No }\end{array}$ & Sex & Age & Symptoms & $\begin{array}{l}\text { Clinical } \\
\text { Findings }\end{array}$ & $\begin{array}{l}\text { Imaging } \\
\text { finding-pre }\end{array}$ & $\begin{array}{l}\text { Imaging } \\
\text { findings- } \\
\text { post }\end{array}$ & $\begin{array}{l}\text { Comor } \\
\text { bidity }\end{array}$ & $\begin{array}{l}\text { Lesion } \\
\text { location }\end{array}$ & $\begin{array}{c}\text { Stenosis } \\
\%\end{array}$ & $\begin{array}{l}\text { Stent } \\
\text { Taxus } \\
(\mathrm{mm})\end{array}$ & $\begin{array}{l}\text { 6th } \\
\text { Month } \\
\text { Control } \\
\text { Angio }\end{array}$ & $\begin{array}{c}\text { 1st } \\
\text { Year } \\
\text { Control } \\
\text { CTA }\end{array}$ & $\begin{array}{l}\text { 2nd } \\
\text { Year } \\
\text { control } \\
\text { Angio }\end{array}$ & $\begin{array}{l}\text { Other } \\
\text { stented area/ } \\
\text { stenosis } \\
\text { Additional } \\
\text { findings }\end{array}$ & Complication \\
\hline 9 & M & 54 & R Hp, D & $\begin{array}{c}\text { (Previous } \\
\text { CVE) } \\
\text { RUE, RLE } \\
\text { 2/5, LUE, LLE } \\
\text { 3/5, } \\
\text { dysphasia }\end{array}$ & $\begin{array}{l}\text { MRI: Left MCA } \\
\text { area- chronic } \\
\text { large hemorrhagic } \\
\text { infarct, Wallerian } \\
\text { degeneration }\end{array}$ & - & $\mathrm{DM}, \mathrm{C}$ & RVA & $50-70$ & $3 \times 12$ & $\mathrm{MIH}$ & $P$ & BTA: P & $\begin{array}{c}\text { (L ICA } \\
\text { occlusion } \\
\text { RVA stenosis- } \\
\text { follow-up }\end{array}$ & - \\
\hline 10 & M & 63 & $\mathrm{Hd}$ & - & - & - & CAD & LVA & $>90$ & $4.5 \times 12$ & ISR & - & - & $\begin{array}{l}\text { L ICA }>\% 90 \\
\text { (R ICA, RVA } \\
\text { occlusion) }\end{array}$ & - \\
\hline 11 & M & 81 & $\begin{array}{c}\mathrm{N}, \mathrm{Hd}, \mathrm{R} \\
\mathrm{Hp}\end{array}$ & $\begin{array}{l}\text { CVE; Opens } \\
\text { his eyes } \\
\text { to verbal } \\
\text { stimulus, } \\
\text { head } \\
\text { movements } \\
\text { to the } \\
\text { questions, } \\
\text { localizes the } \\
\text { pain with } \\
\text { L hand, L } \\
\text { arm, leg } \\
\text { spontaneous } \\
\text { movement, } \\
\text { R extremity } \\
\text { extantion??? }\end{array}$ & $\begin{array}{l}\text { MRI: Bilateral } \\
\text { cerebellar, tectal, } \\
\text { R hipocampal, } \\
\text { bilateral occipital } \\
\text { acute ischemic } \\
\text { lesions }\end{array}$ & - & - & LVA & $50-70$ & $4.5 \times 12$ & - & - & - & $\begin{array}{c}\text { (BA } \\
\text { thrombolysis) }\end{array}$ & - \\
\hline 12 & M & 66 & V, D, At & $\begin{array}{c} \\
\text { (Previous } \\
\text { CVE) } \\
\text { Ataxic walk, } \\
\text { bilateral } \\
\text { intantional } \\
\text { tremor, } \\
\text { Romberg+ }\end{array}$ & $\begin{array}{l}\text { MRI: A couple } \\
\text { milimetric } \\
\text { noncpesific } \\
\text { lesion in L frontal } \\
\text { subcortical white } \\
\text { matter } \\
\text { CTA: Intimal } \\
\text { hyperplasia } \\
\text { and mechanical } \\
\text { complication } \\
\text { (broken stent) } \\
\text { likely to cause } \\
\text { hemodynamically } \\
\text { significant } \\
\text { stenosis in L } \\
\text { vertebral ostial } \\
\text { stent }\end{array}$ & - & DM & $\begin{array}{l}\text { LVA } \\
\text { Broken } \\
\text { stent }\end{array}$ & - & $4.5 \times 16$ & $P$ & - & - & $\begin{array}{c}\text { RVA } \\
\text { occlusion }\end{array}$ & - \\
\hline 13 & W & 54 & $\mathrm{NV}, \mathrm{V}, \mathrm{At}$ & $\begin{array}{l}\text { (Previous } \\
\text { CVE) } \\
\text { Ataxic } \\
\text { walk, RUE } \\
\text { movement } \\
\text { and } \\
\text { cerebellar } \\
\text { examination } \\
\text { awkward }\end{array}$ & $\begin{array}{c}\text { CT, CTA: } R \\
\text { cerebellar enfarct, } \\
\text { L ICA }>50 \% \\
\text { stenosis }\end{array}$ & - & DM & $\begin{array}{l}\text { RVA } \\
\text { LVA }\end{array}$ & $\begin{array}{l}>90 \\
>90\end{array}$ & $\begin{array}{l}5 \times 12 \\
5 \times 16\end{array}$ & $\begin{array}{c}\mathrm{P} \\
\mathrm{MIH}\end{array}$ & $\begin{array}{c}\mathrm{P} \\
\mathrm{MIH}\end{array}$ & - & $\begin{array}{c}\text { (L ICA } \\
\text { stenosis- } \\
\text { follow-up) }\end{array}$ & - \\
\hline 14 & $M$ & 64 & $\begin{array}{c}\text { Temporary } \\
\text { D }\end{array}$ & $\begin{array}{l}\text { (Previous } \\
\text { CVE) } \\
-\end{array}$ & - & - & $\begin{array}{l}\mathrm{DM}, \\
\mathrm{Ht}, \mathrm{C} \\
\mathrm{CAD}\end{array}$ & LVA & $50-70$ & $5 \times 12$ & $\mathrm{MIH}$ & $\mathrm{MIH}$ & - & $\begin{array}{c}\text { (L ICA } \\
\text { occlusion } \\
\text { RICA } \\
\text { stenosis- } \\
\text { follow-up) }\end{array}$ & - \\
\hline 15 & M & 67 & $\begin{array}{c}\text { S, } \\
\text { temporary } \\
\text { visual, } \\
\text { hearing } \\
\text { loss }\end{array}$ & $\begin{array}{l}\text { (Previous } \\
\text { CVE) } \\
-\end{array}$ & - & - & $\mathrm{Ht}$ & LVA & $>90$ & $4.5 \times 12$ & $\mathrm{MIH}$ & P & - & $\begin{array}{l}\text { (R ICA, L ICA } \\
\text { stenosis- } \\
\text { follow-up) }\end{array}$ & - \\
\hline 16 & M & 66 & N-L arm & - & $\begin{array}{l}\text { MRI: bulbus } \\
\text { posterior R sided } \\
\text { hyperintense } \\
\text { milimetric lesions } \\
\text { possibly due to } \\
\text { chronic ischemia } \\
\text { CTA: Soft plaques } \\
\text { that do not } \\
\text { cause stenosis in } \\
\text { bilateral carotid } \\
\text { bifurcation, L } \\
\text { distal CCA }\end{array}$ & - & $\mathrm{Ht}, \mathrm{DM}$ & LVA & $>90$ & $4 \times 16$ & $\mathrm{MIH}$ & P & - & $\mathrm{RVA}>90$ & - \\
\hline
\end{tabular}




\section{Table 1. Patients demographics, findings and treatments (continued)}

\begin{tabular}{|c|c|c|c|c|c|c|c|c|c|c|c|c|c|c|c|}
\hline $\begin{array}{l}\text { Case } \\
\text { No }\end{array}$ & Sex & Age & Symptoms & $\begin{array}{l}\text { Clinical } \\
\text { Findings }\end{array}$ & $\begin{array}{l}\text { Imaging } \\
\text { finding-pre }\end{array}$ & $\begin{array}{l}\text { Imaging } \\
\text { findings- } \\
\text { post }\end{array}$ & $\begin{array}{l}\text { Comor } \\
\text { bidity }\end{array}$ & $\begin{array}{l}\text { Lesion } \\
\text { location }\end{array}$ & $\begin{array}{c}\text { Stenosis } \\
\%\end{array}$ & $\begin{array}{l}\text { Stent } \\
\text { Taxus } \\
(\mathrm{mm})\end{array}$ & $\begin{array}{l}\text { 6th } \\
\text { Month } \\
\text { Control } \\
\text { Angio }\end{array}$ & $\begin{array}{c}\text { 1st Year } \\
\text { Control } \\
\text { CTA }\end{array}$ & $\begin{array}{l}\text { 2nd } \\
\text { Year } \\
\text { control } \\
\text { Angio }\end{array}$ & $\begin{array}{l}\text { Other stented } \\
\text { area/stenosis } \\
\text { Additional } \\
\text { findings }\end{array}$ & Complication \\
\hline 17 & M & 47 & $\begin{array}{l}\mathrm{V}, \mathrm{NV}, \mathrm{Vd}, \\
\mathrm{Di}, \mathrm{At}\end{array}$ & $\begin{array}{l}\text { CVE: double } \\
\text { vision at } \\
\text { left glance, } \\
\text { nistagmus, } \\
\text { quadranopsia, } \\
\text { obliterated } \\
\text { L nasolabial } \\
\text { fold, At to the } \\
\text { L, minimally } \\
\text { awkward } \\
\text { knee heel test }\end{array}$ & $\begin{array}{l}\text { MRI: LPCA } \\
\text { area, bilateral } \\
\text { talamic, } \\
\text { upper pons, } \\
\text { bilateral } \\
\text { serebellar } \\
\text { acute infact } \\
\text { areas } \\
\text { CTA: LVa } \\
\text { occlusion } 4 \\
\text { cm distal to } \\
\text { the origin, } \\
\text { no prebasilar } \\
\text { segment, } \\
\text { thin, irregular } \\
\text { basilar } \\
\text { artery, LPCA } \\
\text { occlusion }\end{array}$ & - & $C$ & LVA & $70-90$ & $4.5 \times 16$ & $\mathrm{MIH}$ & $P$ & - & $\begin{array}{l}\text { (Hypoplastic } \\
\text { RVA) }\end{array}$ & - \\
\hline 18 & M & 70 & $E A, V$ & - & $\begin{array}{l}\text { MRI: No } \\
\text { acute } \\
\text { ischemic } \\
\text { lesion }\end{array}$ & - & $\mathrm{Ht}$ & RVA & $>90$ & $4 \times 16$ & $P$ & - & - & $\begin{array}{c}\text { R ICA }>90 \\
\text { (LVA, L subclav, } \\
\text { L ICA stenosis- } \\
\text { follow-up) }\end{array}$ & - \\
\hline 19 & M & 53 & V, NV, S & - & $\begin{array}{c}\text { MRI: A } \\
\text { couple } \\
\text { milimetric } \\
\text { nonspesific } \\
\text { gliotic lesion } \\
\text { in bilateral } \\
\text { subcortical } \\
\text { area }\end{array}$ & - & C & LVA & $70-90$ & $4 \times 16$ & $\mathrm{MIH}$ & $P$ & - & $\begin{array}{l}\text { (RVA stenosis- } \\
\text { follow-up) }\end{array}$ & $\begin{array}{l}\text { L subclav } \\
\text { dissection }\end{array}$ \\
\hline 20 & W & 72 & $\begin{array}{l}\text { Hd, V, D, } \\
\text { N-L arm }\end{array}$ & - & $\begin{array}{c}\text { MRI: L } \\
\text { cerebellar } \\
\text { chronic } \\
\text { ischemic } \\
\text { lesion. } \\
\text { Milimetric } \\
\text { ischemic } \\
\text { gliotic } \\
\text { lesions in } \\
\text { cerebral } \\
\text { subcortikal } \\
\text { white matter }\end{array}$ & - & $\mathrm{Ht}$ & RVA & $>90$ & $4 \times 12$ & $\mathrm{MIH}$ & - & - & $\begin{array}{c}\text { R ICA \%70-90 } \\
\text { RVA cervical } \\
\% 50-70 \\
\text { (LVA occlusion) }\end{array}$ & - \\
\hline 21 & M & 62 & V & - & $\begin{array}{l}\text { MRI: No } \\
\text { acute } \\
\text { ischemic } \\
\text { lesion. } \\
\text { Bilateral } \\
\text { milimetric } \\
\text { chronic } \\
\text { ischemic } \\
\text { lesions }\end{array}$ & - & DM & RVA & $70-90$ & $4 \times 12$ & $P$ & $P$ & - & $\begin{array}{l}\text { (RVA intradural } \\
\text { stenosis } \\
\text { Milim ACoA } \\
\text { aneurism- } \\
\text { follow-up) }\end{array}$ & - \\
\hline 22 & M & 55 & $\begin{array}{l}\mathrm{V}, \mathrm{Vd}, \mathrm{N}-\mathrm{L} \\
\text { face }\end{array}$ & - & $\begin{array}{l}\text { MRI: No } \\
\text { acute } \\
\text { ischemic } \\
\text { lesion }\end{array}$ & - & $\begin{array}{l}\mathrm{Ht}, \mathrm{HL} \\
\text { COPD }\end{array}$ & LVA & $>90$ & $4.5 \times 16$ & $\mathrm{MIH}$ & - & - & $\begin{array}{l}\text { L ICA occlusion- } \\
\text { reconstruction } \\
\text { with cervical } \\
\text { collateral }\end{array}$ & - \\
\hline 23 & $M$ & 65 & $\begin{array}{l}\text { Previous } \\
\text { TIA,V }\end{array}$ & - & $\begin{array}{l}\text { MRI: No } \\
\text { acute } \\
\text { ischemic } \\
\text { lesion. L } \\
\text { frontal } \\
\text { chronic } \\
\text { enfarct. } \\
\text { Lacunar } \\
\text { enfarct in } \\
\text { L caudat } \\
\text { nucleus }\end{array}$ & - & C, CAD & RVA & $70-90$ & $4 \times 16$ & $P$ & - & - & $\begin{array}{l}\text { RVA intracran } \\
\text { stenosis stent, } \\
\text { R PICA origin } \\
\text { aneurism coil } \\
\text { emb }\end{array}$ & - \\
\hline 24 & $M$ & 44 & $\begin{array}{l}\text { V, N-L } \\
\text { arm- lip }\end{array}$ & - & $\begin{array}{l}\text { MRI: No } \\
\text { acute } \\
\text { ischemic } \\
\text { lesion. A } \\
\text { couple } \\
\text { millimetric } \\
\text { nonspesific } \\
\text { lesion }\end{array}$ & - & $\begin{array}{l}\mathrm{Ht}, \\
\text { renal } \\
\text { artery } \\
\text { stent }\end{array}$ & RVA & $70-90$ & $4 \times 16$ & $\mathrm{MIH}$ & - & - & - & - \\
\hline
\end{tabular}


During the early postoperative period within the seven days, one patient (case 1) had vomiting twice with a fifteen seconds long asystole. ECG and cardiac enzymes were normal with no additional problems.

One patient (case 11) succumbed to death due to aspiration pneumonia 11 days after the procedure. The patient was brought to the hospital unconscious with a basilar artery stroke. Additional left VA stenosis was diagnosed and treated with PES during the same session of angiography for intraarterial thrombolysis. The patient benefited from intraarterial thrombolysis, neurological symptoms regressed and was extubated. Nevertheless, he couldn't survive.

\section{Follow-up}

The mean follow-up was 447 days, the median follow-up was 399 days.

The follow-up findings are summarized in table 2. Twentythree of the 24 patients with a total of 27 PES were evaluated at the 6th month (median 187 days) with DSA. Only one stent (case 10) out of 27 stents was diagnosed with hemodynamically significant ISR (3.7\%). The patient was a 63-year-old male, diagnosed with a right $V A$ and ICA occlusion with $90 \%$ stenosis of left VA and ICA during cardiology controls. Left VA and left ICA stenoses were treated with endovascular stenting. At the 6th month follow-up, there was MIH in the left ICA stent but hemodynamically significant ( $>50 \%$ ) ISR in the left VA stent (Fig. 2). Meanwhile, the patient stopped using the ASA but continued to use clopidogrel due to stomach bleeding. We planned a treatment session of balloon angioplasty, unfortunately, the patient refused the treatment due to an additional recent diagnosis of lung carcinoma. Furthermore, he didn't continue follow-ups and died of complications of lung carcinoma.
At the first and second-year evaluations, no other hemodynamically significant ISR were investigated.

\begin{tabular}{|c|c|c|c|c|c|c|c|c|c|c|}
\hline \multicolumn{1}{|c|}{ Table 2. Follow-up findings } \\
\hline Month & $\begin{array}{c}\text { Medi- } \\
\text { an } \\
\text { day }\end{array}$ & $\begin{array}{c}\text { Pati- } \\
\text { ent } \\
(\mathrm{n})\end{array}$ & $\begin{array}{c}\text { Stent } \\
(\mathrm{n})\end{array}$ & $\begin{array}{c}\text { DSA/ } \\
\text { CTA }\end{array}$ & $\begin{array}{c}\text { Patent } \\
(\mathrm{n})\end{array}$ & $\begin{array}{c}\text { Patent } \\
(\%)\end{array}$ & $\begin{array}{c}\text { MIH } \\
(\mathrm{n})\end{array}$ & $\begin{array}{c}\text { MIH } \\
(\%)\end{array}$ & $\begin{array}{c}\text { ISR } \\
(\mathrm{n})\end{array}$ & $\begin{array}{c}\text { ISR } \\
(\%)\end{array}$ \\
\hline 6 & 187 & 23 & 27 & $27 /-$ & 10 & $37 \%$ & 16 & $59 \%$ & 1 & $3.7 \%$ \\
\hline 12 & 370 & 17 & 22 & $2 / 20$ & 18 & $81.80 \%$ & 4 & $18.20 \%$ & - & - \\
\hline 24 & 736 & 7 & 11 & $8 / 3$ & 8 & $72.72 \%$ & 3 & $27.30 \%$ & - & - \\
\hline n: number
\end{tabular}

Only one patient (4.1\%) had recurrent neurological symptoms during the follow-ups (case 12). The patient had recurrent complaints of balance and speech problems. Two months before being referred to our clinic, he was treated with left vertebral ostial stenting in another clinic. The patient had a broken stent and was treated with PES through both segments of the fragmented stent (Fig. 3). The 6th month follow-up after this procedure yielded a normal DSA. 9 months after the second procedure there was worsening of the cerebellar symptoms. Nevertheless, the left VA ostial stent was patent at angiographic controls. No lesion that had restricted diffusion on MRI. No other findings on spinal MRI explained the symptoms. The cerebellar findings were recovered and the patient was discharged after 10 days of hospitalization.

In a median follow-up of 13 months (6-25 months), none of the patients had late stent thrombosis. The procedural and post-procedural events are collected in table 3.

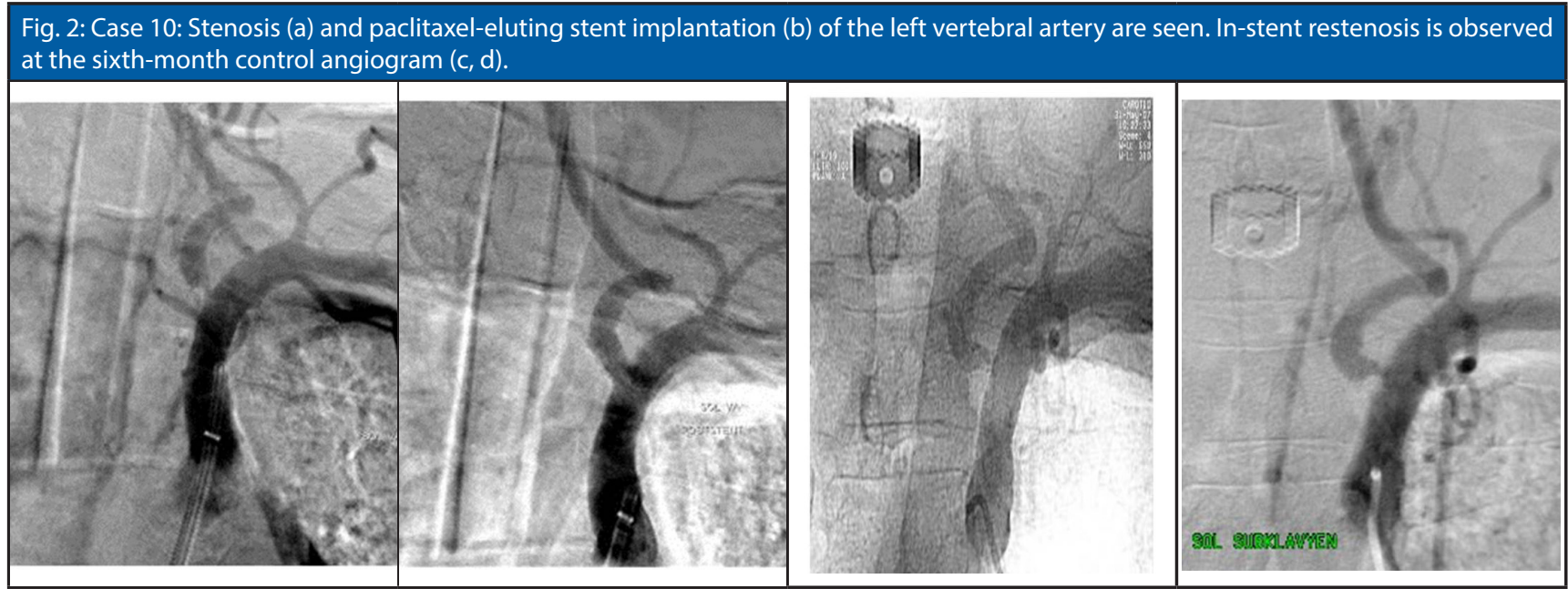




\begin{tabular}{|c|c|c|c|c|c|c|}
\hline \multicolumn{2}{|c|}{ Table 3. Procedural, post-procedural events } & $\begin{array}{c}\text { Major } \\
\text { event }\end{array}$ & $\mathrm{n}$ & $\%$ & Minor event & $\%$ \\
\hline $\begin{array}{c}\text { Procedure } \\
\text { related } \\
\text { complications }\end{array}$ & - & - & - & Limited proximal subclavian artery dissection & 4.1 \\
\hline $\begin{array}{c}30 \text { days } \\
\text { adverse } \\
\text { events }\end{array}$ & $\begin{array}{c}\text { Exitus } \\
\text { due to } \\
\text { aspiration } \\
\text { pnomonia }\end{array}$ & 1 & 4.1 & $\begin{array}{c}\text { Emotional indifference- agitation/ Milimetric hemorrhagic lesion- L caudate nucleus } \\
\text { Vomiting- vagal stimulation, 15 seconds asystole }\end{array}$ & 3 & 12.5 \\
\hline $\begin{array}{c}\text { Long period } \\
\text { events }\end{array}$ & - & - & - & $\begin{array}{c}\text { Temporary numbness on left hand/Two milimetric acute ischemic lesion- R postcentral } \\
\text { Worsening of cerebellar symptoms }\end{array}$ & 4.1 \\
\hline
\end{tabular}

Fig. 3: Case 12: It was observed that the stent, previously placed on the VA origin, was broken (a, b). Angiography images (c, d) obtained after insertion of the paclitaxel-eluting stent through the broken components are observed.

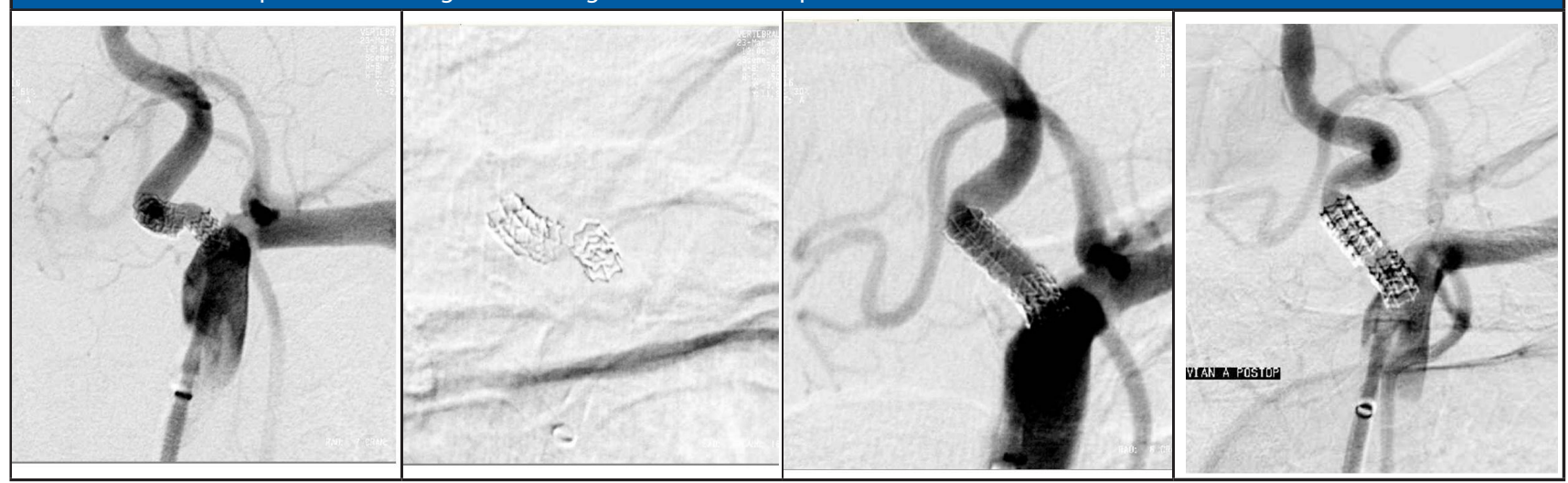

\section{Discussion}

Proximal extracranial VA is the second most common stenotic area after carotid artery bifurcation. The origin of the VA is a difficult region to display (8).

Currently, catheter angiography is the gold standard in the evaluation of the VA origin and plaque, the detection of ulceration and thrombus, and the evaluation of extra and intracranial blood flow (9). However, it includes the risks of invasiveness, hospitalization, ionizing radiation, contrast agent allergy and nephropathy. Therefore, DSA is not the first method of choice in the diagnosis of extracranial VA atherosclerotic disease. Color Doppler Ultrasonography (US) and MRI have some technical and anatomical limitations in VA origin imaging. Spiral and multislice CTA can display extracranial VA without the risks of DSA (10).

We included DSA for the diagnosis of VA stenosis in the study. CTA was alternated with DSA to decrease the risks of DSA for the follow-up of the cases.
Classically the VA ostial atherosclerotic disease is treated with antiplatelet medication and anticoagulation. The benefit of an intervention over medical treatment alone is unclear. Various small trials were conducted to determine the efficiency of endovascular treatment modality relative to medical therapy alone. Nevertheless, these trials failed to demonstrate the superiority of stenting over the best medical therapy (11-13). Large randomized controlled trials are required to elucidate this question.

The generally accepted interventional indications are symptomatic VA stenosis, persistent posterior system ischemic symptoms despite optimal medical therapy, or intolerance to medical therapy. However, published studies may justify attempts without a medical treatment with symptomatic disease (14). The severity of stenosis, angiographic appearance (vulnerability, presence of ulceration), adequacy of collateral flow and patient age are the effective factors for decisions (14). 
In embolic posterior circulation ischemia when cardiac causes are eliminated, the embolic event should be considered primarily due to VA origin disease. In these cases, treatment is recommended even the degree of stenosis is less than $50 \%$, as it is a source of embolism (15).

Asymptomatic patients with significant stenosis in the origin of VA are controversial. Although most of the asymptomatic patients don't require, some researchers suggest endovascular treatment to high grade (more than 70\%) stenosis in dominant VA or single VA origin for the increased risk of embolism (16). In a young asymptomatic patient, endovascular treatment is also recommended for severe ulcerated stenosis without good collateral flow. Another group of researchers argues that asymptomatic patients should be treated in cases where collateral circulation is necessary and is of great importance, such as carotid occlusion (8).

In our hospital, endovascular treatment is recommended for patients with posterior circulation ischemic symptoms despite optimal medical treatment, and those with more than $50 \%$ stenosis in the origin of VA in DSA. In addition, endovascular treatment is recommended to patients with an ulcerated plaque in the origin of $\mathrm{VA}$, regardless of whether they are symptomatic or asymptomatic.

The ostial VA stenoses are highly elastic lesions due to the well-developed muscular layer of VA origin like coronary arteries. Thus, for successful treatment, it is necessary to use stents with high radial force (17). In the SSYLVIA trial, the increased restenosis rates in VA origin lesions compared to the lesions in intracranial vessels and VA segments prior to posterior inferior cerebellar artery have been associated with the lesions' high elastic nature and the stent's design (18).

Primary stenting with balloon-expandable coronary stents were reported to be applied safely with high technical success in VAOS (17). Although percutaneous stenting decreases the rate of procedural complications such as failed balloon dilatation or risk of dissection, the high ISR-rates remain as a problem to be solved $(17,19)$.

In parallel with the data of the decreased ISR-rates of antiproliferative DES in coronary arteries (20), DES were started to be used in VAOS as an alternative. The first-generation DES is sirolimus and PES. It is known that paclitaxel and sirolimus-eluting stents decrease the neointimal hyperplasia by inhibiting the induced smooth muscle proliferation by mitogens (21).
Although some studies revealed no differences in the ISRrate between DES and bare-metal stents (BMS) (22) the results of two metanalyses showed a reduction of the ISRrate and a lower rate of recurrent symptoms- symptomatic restenosis for DES compared with the BMS group (23, 24). It is indicated that the relative ISR problem remains relevant $(24,25)$. The prospective randomized STOVAST trial revealed no evidence for ISR reduction with DES versus BMS group (26). In this trial, cobalt-chromium stents in the BMS group were observed to have the lowest ISR-rates (8.3\%, $1 / 12$ patients) whereas the PES in the DES group was observed to have the highest ISR-rate $(50 \%, 2 / 4$ patients). Our data differ from the STOVAST trial significantly. We have found only $3.7 \%$ (only one asymptomatic case among 27 stent applications) hemodynamically significant (which is $>50 \%$ ) ISR-rate occurred in about 6 months after stenting with PES in our retrospective study within a mean of 447 days of follow-up. The patient had a combined contralateral ICA and VA occlusion. In a recent study, the contralateral VA occlusion at the time of stenting is postulated as an increased risk factor for ISR (27). The differences between the reported restenosis rates may be connected with the number of patients, the median interval follow-up time and the differences of the evaluation methods as well as application-related differences.

Although DESs are being used in the supra-aortic and intracranial vessels, the long period results are not clear. The late and very late thrombosis remains as a problem. In the light of these data and considering the costs, as of today, DESs are preferred to be used in the ostial VA stenosis for stent-in-stent placement for ISR of BMS and/or broken stents.

Treatment protocols of VAOS still maintain their dynamism. Recently, the use of distal protection devices and the self-expandable stents for the treatment of ostial VA stenosis have been investigated $(28,29)$. To define the best therapy option, prospective studies with long-term results are required.

We assessed the technical success, efficiency, clinical and angiographic results of PES in vertebral ostial stenosis. Despite the shortcomings of a retrospective study, we showed that VAOS can be treated effectively and safely with high technical success and low ISR-rates with paclitaxel DESs. With low restenosis rates, the antiproliferative DESs are still an alternative for reducing the VA ISR. 


\section{REFERENCES}

1. Bamford J, Sandercock P, Dennis M, Burn J, Warlow C. Classification and natural history of clinically identifiable subtypes of cerebral infarction. Lancet 1991;337(8756):1521-1526.

2. Phatouros CC, Higashida RT, Malek AM, Meyers PM, Lefler JE, Dowd $\mathrm{CF}$, Halbach VV. Endovascular treatment of noncarotid extracranial cerebrovascular disease. Neurosurgery clinics of North America 2000;11(2):331-350.

3. Hass WK, Fields WS, North RR, Kircheff, II, Chase NE, Bauer RB. Joint study of extracranial arterial occlusion. II. Arteriography, techniques, sites, and complications. Jama 1968;203(11):961-968.

4. Cloud GC, Markus HS. Diagnosis and management of vertebral artery stenosis. QJM : monthly journal of the Association of Physicians 2003;96(1):27-54.

5. Jenkins JS, White CJ, Ramee SR, Collins TJ, Chilakamarri VK, McKinley KL, Jain SP. Vertebral artery stenting. Catheterization and cardiovascular interventions : official journal of the Society for Cardiac Angiography \& Interventions 2001;54(1):1-5.

6. Jenkins JS, White CJ, Ramee SR, Collins TJ, McKinley KL. Vertebral Insufficiency: When to Intervene and How? Current interventional cardiology reports 2000;2(2):91-94.

7. 7. North American Symptomatic Carotid Endarterectomy Trial. Methods, patient characteristics, and progress. Stroke 1991;22(6):711-720.

8. Henry M, Henry I, Klonaris C, al. e. Angioplasty and stenting of the carotid and supra-aortic trunks: Percutaneous transluminal angioplasty and stenting of extracranial VA stenosis. 1st ed. London, UK: Taylor and Francis Medicine, 2003.

9. Berteloot D, Leclerc X, Leys D, Krivosic R, Pruvo JP. [Cerebral angiography: a study of complications in 450 consecutive procedures]. Journal de radiologie 1999;80(8):843-848.

10. Omori $Y$, Komatsu S, Murakawa T, Hirayama A, Sato Y, Fujisawa $\mathrm{Y}$, Higashide T, Takahashi M, Kodama K. MDCT detection of left subclavian artery obstruction accompanied by anomalous origin of the left vertebral artery. International journal of cardiology 2007;115(2):244-245. doi: 10.1016/j.ijcard.2006.01.055

11. Coward LJ, McCabe DJ, Ederle J, Featherstone RL, Clifton A, Brown MM. Long-term outcome after angioplasty and stenting for symptomatic vertebral artery stenosis compared with medical treatment in the Carotid And Vertebral Artery Transluminal Angioplasty Study (CAVATAS): a randomized trial. Stroke 2007;38(5):1526-1530. doi: 10.1161/STROKEAHA.106.471862

12. Compter A, van der Worp HB, Schonewille WJ, Vos JA, Boiten J, Nederkoorn PJ, Uyttenboogaart M, Lo RT, Algra A, Kappelle LJ. Stenting versus medical treatment in patients with symptomatic vertebral artery stenosis: a randomised open-label phase 2 trial. The Lancet Neurology 2015;14(6):606-614. doi: 10.1016/ S1474-4422(15)00017-4

13. Markus HS, Larsson SC, Kuker W, Schulz UG, Ford I, Rothwell PM, Clifton A. Stenting for symptomatic vertebral artery stenosis: The Vertebral Artery Ischaemia Stenting Trial. Neurology 2017;89(12):1229-1236. doi: 10.1212/WNL.0000000000004385

14. Mukherjee D, Rosenfeld K. Manual of peripheral vascular intervention: Vertebral artery disease. Philadelphia, 2005.

15. Piotin M, Spelle L, Martin JB, Weill A, Rancurel G, Ross IB, Rufenacht DA, Chiras J. Percutaneous transluminal angioplasty and stenting of the proximal vertebral artery for symptomatic stenosis. AJNR American journal of neuroradiology 2000;21(4):727-731.

16. Wehman JC, Hanel RA, Guidot CA, Guterman LR, Hopkins LN. Atherosclerotic occlusive extracranial vertebral artery disease: indications for intervention, endovascular techniques, shortterm and long-term results. Journal of interventional cardiology 2004;17(4):219-232. doi: 10.1111/j.1540-8183.2004.04055.x
17. Lin $\mathrm{YH}$, Juang JM, Jeng JS, Yip PK, Kao HL. Symptomatic ostial vertebral artery stenosis treated with tubular coronary stents:clinical results and restenosis analysis. Journal of endovascular therapy : an official journal of the International Society of Endovascular Specialists 2004;11(6):719-726. doi: 10.1583/04-1336.1

18. Stenting of Symptomatic Atherosclerotic Lesions in the Vertebral or Intracranial Arteries (SSYLVIA): study results. Stroke 2004;35(6):1388-1392. doi: 10.1161/01.STR.0000128708.86762.d6

19. Lin YH, Liu YC, Tseng WY, Juang JM, Hung CS, Lin JW, Jeng JS, Yip $\mathrm{PK}, \mathrm{KaO} \mathrm{HL}$. The impact of lesion length on angiographic restenosis after vertebral artery origin stenting. European journal of vascular and endovascular surgery : the official journal of the European Society for Vascular Surgery 2006;32(4):379-385. doi: 10.1016/j. ejvs.2006.02.016

20. Morice MC, Serruys PW, Sousa JE, Fajadet J, Ban Hayashi E, Perin M, Colombo A, Schuler G, Barragan P, Guagliumi G, Molnar F, Falotico $R$. A randomized comparison of a sirolimus-eluting stent with a standard stent for coronary revascularization. The New England journal of medicine 2002;346(23):1773-1780. doi: 10.1056/ NEJMoa012843

21. Axel DI, Kunert W, Goggelmann C, Oberhoff $M$, Herdeg C, Kuttner A, Wild DH, Brehm BR, Riessen R, Koveker G, Karsch KR. Paclitaxel inhibits arterial smooth muscle cell proliferation and migration in vitro and in vivo using local drug delivery. Circulation 1997;96(2):636-645.

22. Raghuram K, Seynnaeve C, Rai AT. Endovascular treatment of extracranial atherosclerotic disease involving the vertebral artery origins: a comparison of drug-eluting and bare-metal stents. Journal of neurointerventional surgery 2012;4(3):206-210. doi: 10.1136/neurintsurg-2011-010051

23. Tank VH, Ghosh R, Gupta V, Sheth N, Gordon S, He W, Modica SF, Prestigiacomo CJ, Gandhi CD. Drug eluting stents versus bare metal stents for the treatment of extracranial vertebral artery disease: a meta-analysis. Journal of neurointerventional surgery 2016;8(8):770-774. doi: 10.1136/neurintsurg-2015-011697

24. Langwieser N, Buyer D, Schuster T, Haller B, Laugwitz KL, Ibrahim T. Bare metal vs. drug-eluting stents for extracranial vertebral artery disease: a meta-analysis of nonrandomized comparative studies. Journal of endovascular therapy : an official journal of the International Society of Endovascular Specialists 2014;21(5):683692. doi: 10.1583/14-4713MR.1

25. Musialek P, Langwieser N. Commentary: vertebral artery ostial stenosis stenting technique: the concept reversed? Journal of endovascular therapy : an official journal of the International Society of Endovascular Specialists 2015;22(3):445-448. doi: $10.1177 / 1526602815583490$

26. http://spo.escardio.org/eslides/view.aspx?eevtid $=54 \& f p=3170$.

27. Li J, Hua Y, Needleman L, Forsberg F, Eisenbray JR, Li Z, Liu $R$, Tian $X$, Jiao $L$, Liu JB. Arterial occlusions increase the risk of in-stent restenosis after vertebral artery ostium stenting. Journal of neurointerventional surgery 2018. doi: 10.1136/ neurintsurg-2018-014243

28. Geng X, Hussain M, Du H, Zhao L, Chen J, Su W, Ma L, Gao Z, Ding $Y$, Ji X. Comparison of self-expanding stents with distal embolic protection to balloon-expandable stents without a protection device in the treatment of symptomatic vertebral artery origin stenosis: a prospective randomized trial. Journal of endovascular therapy : an official journal of the International Society of Endovascular Specialists 2015;22(3):436-444. doi: 10.1177/1526602815579406

29. Chung SY, Lee DH, Choi JW, Choi BS, In HS, Kim SM, Choi CG, Kim SJ, Suh DC. Use of self-expanding stents for the treatment of vertebral artery ostial stenosis: a single center experience. Korean journal of radiology 2010;11(2):156-163. doi: 10.3348/kjr.2010.11.2.156 